\title{
InterCriteria Analysis of Multi-population Genetic Algorithms Performance
}

\author{
Maria Angelova, Tania Pencheva \\ Institute of Biophysics and Biomedical Engineering \\ Bulgarian Academy of Sciences \\ 105 Acad. G. Bonchev Str., Sofia 1113, Bulgaria \\ Emails: maria.angelova@biomed.bas.bg, tania.pencheva@biomed.bas.bg
}

\begin{abstract}
InterCriteria Analysis approach is here applied for the assessment of promising genetic algorithms optimization techniques. Altogether six multi-population genetic algorithms are here considered, differing in the execution order of main genetic operators selection, crossover and mutation. InterCriteria Analysis approach, based on the apparatuses of index matrices and intuitionistic fuzzy sets, is implemented to assess the performance of multi-population genetic algorithms for the parameter identification of Saccharomyces cerevisiae fed-batch fermentation process. Degrees of "agreement" and "disagreement" between the algorithms outcomes convergence time and model accuracy, from one hand, and model parameters estimations, from the other hand, have been established. Outlined relations are going to lead to an additional exploring of the model, expected to be extraordinary valuable especially in the case of modelling of living systems, such as fermentation processes.
\end{abstract}

\section{INTRODUCTION}

$\mathbf{Y}$ EAST is widely used model organisms in contemporary biotechnology and genetic engineering due to its well known metabolic pathways [1], [15]. Specifically, $S$. cerevisiae has found significant application in the production of medicines, food and beverages. Meanwhile, the complex structure of fermentation processes, usually described by systems of non-linear differential equations with several specific growth rates, turns their modelling in a challenging and rather difficult task.

Genetic algorithms (GA) is a stochastic global optimization technique, proven in successful solving of a variety of challenging problems in the field of complex dynamic systems optimization [9], [10], [16], among them for parameter identification of various fermentation process models [1], [3], [14], [15], [16]. GA are one of the methods based on biological evolution, inspired by Darwins theory of survival of the fittest. Simple genetic algorithms, originally presented in [10], search a global optimal solution using three main genetic operators in a sequence selection, crossover and mutation over the individuals in one population. Meanwhile, multipopulation genetic algorithms (MpGA) is more similar to the nature since in it many populations, called subpopulations, evolve independently from each other. After a certain number of generations, a part of individuals migrates between the subpopulations.

The main purpose of this investigation is to be assessed the algorithms performance of MpGA modifications with different sequence of implementation of the main genetic operators, namely selection, crossover and mutation. InterCriteria Analysis ( ICrA), developed as an alternative to the traditional methods for the assessment of algorithms performance, is here implemented. So far, there are some successful ICrA application for parameter identification of fermentation process models [2], [12], [13], [17], where the approach applicability has been demonstrated for establishing correlations between model parameters and GA objective function value and convergence time, from one side, and model parameters themselves, from the other side. This investigation is focussed on the assessment of six MpGA modifications, consequently applied to parameter identification of a $S$. cerevisiae fed batch fermentation process.

\section{PROBLEM FORMULATION}

Parameter identification of a considered here $S$. cerevisiae fed-batch fermentation process model is performed using real data from on-line and off-line measurements, carried out in the Institute of Technical Chemistry, Hanover, Germany. The details about the process conditions and experimental data set could be found in [15].

According to the mass balance and considering mixed oxidative functional state [15], non-linear mathematical model of $S$. cerevisiae fed-batch fermentation process is commonly described as follows:

$$
\begin{gathered}
\frac{d X}{d t}=\left(\mu_{2 S} \frac{S}{S+k_{S}}+\mu_{2 E} \frac{E}{E+k_{E}}\right) X-\frac{F_{i n}}{V} X \\
\frac{d S}{d t}=-\frac{\mu_{2 S}}{Y_{S X}} \frac{S}{S+k_{S}} X+\frac{F_{\text {in }}}{V}\left(S_{i n}-S\right) \\
\frac{d E}{d t}=-\frac{\mu_{2 E}}{Y_{E X}} \frac{E}{E+k_{E}} X+\frac{F_{\text {in }}}{V} E \\
\frac{d V}{d t}=F_{\text {in }}
\end{gathered}
$$

where $X$ is the biomass concentration, $[\mathrm{g} / \mathrm{l}] ; S$ - substrate concentration, $[\mathrm{g} / \mathrm{l}] ; E$ - ethanol concentration, $[\mathrm{g} / \mathrm{l}] ; F_{\text {in }}$ - feeding rate, $[1 / \mathrm{h}] ; V$ - bioreactor volume, [1]; $S_{\text {in }}$ substrate concentration in the feeding solution, $[\mathrm{g} / \mathrm{l}] ; \mu_{2 S}, \mu_{2 E}$ - maximum values of the specific growth rates, $[1 / \mathrm{h}] ; k_{S}, k_{E}-$ saturation constants, [g/l]; $Y_{S X}, Y_{E X}$ - yield coefficients, [-]. All functions are continuous and differentiable and all model parameters fulfil the requirement for non-zero division. 
For the considered here model (1)-(4), the vector $p=$ $\left[\mu_{2 S}, \mu_{2 E}, k_{S}, k_{E}, Y_{S X}, Y_{E X}\right]$, including six model parameters should be identified.

Mean square deviation between the model output and the experimental data for the process variables biomass, substrate and ethanol, has been used as an optimization criterion:

$$
\begin{array}{r}
J=\sum_{i=1}^{m}\left(X_{\exp }(i)-X_{\text {mod }}(i)\right)^{2}+\sum_{i=1}^{n}\left(S_{\exp }(i)-S_{\text {mod }}(i)\right)^{2}+ \\
\sum_{i=1}^{l}\left(E_{\exp }(i)-E_{\text {mod }}(i)\right)^{2} \rightarrow \min
\end{array}
$$

where $m, n$ and $l$ are the dimensions of the experimental data; $X_{\text {exp }}, S_{\text {exp }}, E_{\text {exp }}, X_{\text {mod }}, S_{\text {mod }}$ and $E_{\text {mod }}$ are, respectively, experimental and model predicted data for biomass, substrate and ethanol.

\section{Multi-POPUlation Genetic AlgORIthMS FOR PARAMETER IDENTIFICATION OF $S$. cerevisiae FED-BATCH FERMENTATION PROCESS}

Genetic algorithms, firstly proposed by Holland [11] and later upgraded by Goldberg [10], are a stochastic searching tool inspired by processes of natural evolution. Frequently used as an alternative to the conventional optimization techniques, both simple and multi-population GA have been successfully applied for different problems solving [9], [10], [15], [16], but multi-population GA is more similar to nature than simple GA. Standard multi-population GA works with many populations of coded parameter sets, called subpopulations and searches a global optimal solution using the main genetic operators in a sequence selection, crossover and mutation. For brevity, this algorithm is here denoted as MpGA-SCM, coming from the operators execution order selection, crossover, mutation. MpGA-SCM starts with a creation of $k$ randomly generated subpopulations, each of them with $n$ chromosomes. After that each chromosome in the subpopulation is evaluated and assigned a fitness value. According to the fitness function, the most suitable solutions are selected. Then, crossover and mutation proceed to form a new offspring. After certain number of generations, called isolation time, individuals migrate between the subpopulations. For the purposes of this investigation, MpGA-SCM terminates when a certain number of generations is reached.

While the main idea of GA is to imitate the processes occurring in nature, one can assume that the probability crossover to come first and then mutation is comparable to that both processes to occur in a reverse order; or selection to be performed before or after crossover and mutation, no matter of their order. Following this idea, five modifications of MpGA-SCM, with different sequence of execution of main genetic operators, have been developed aiming to improve model accuracy and algorithms convergence time [1], [3], [14]. The modifications, namely MpGA-SMC (selection, mutation, crossover), MpGACMS (crossover, mutation, selection), MpGA-MCS (mutation, crossover, selection), MpGA-CSM (crossover, selection, mutation) and MpGA-MSC (mutation, selection, crossover) have been proposed and basically investigated for parameter identification of a fed-batch cultivation of $S$. cerevisiae in [1], [3], [14]. The performance of mentioned above altogether six MpGA, applied to the parameter identification of a $S$. cerevisiae fed-batch cultivation process, is going to be assessed by promising ICrA approach.

\section{INTERCRITERIA ANALYSIS}

The theoretical framework of the InterCriteria Analysis approach, based on the apparatuses of index matrices (IM) [5], [6], [7] and intuitionistic fuzzy sets (IFS) [4], is given in details in [8]. Here, ICrA is briefly presented for a completeness.

The initial IM $A$ in a form presented in (6) consists of the criteria $C_{p}$ (for rows), objects $O_{q}$ (for columns) and real number elements $a_{C_{p}, O_{q}}$ for every $p, q,(1 \leq p \leq m, 1 \leq q \leq n)$.

Further, an IM with index sets consisting of the criteria (for rows and for columns) with IF pair elements determining the degrees of "correspondence" between the respective criteria is constructed. A real number $a_{C_{p}, O_{q}}$ is comparable about relation $R$ with the other $a$-object, so that $R\left(a_{C_{k}, O_{i}}, a_{C_{k}, O_{j}}\right)$ is defined for each $i, j, k$. Let $\bar{R}$ be the dual relation of $R$ in the sense that if $R$ is satisfied, then $\bar{R}$ is not satisfied, and vice versa. For example, if " $R$ " is the relation "<", then $\bar{R}$ is the relation " $>$ ", and vice versa. If $S_{k, l}^{\mu}$ is the number of cases in which $R\left(a_{C_{k}, O_{i}}, a_{C_{k}, O_{j}}\right)$ and $R\left(a_{C_{l}, O_{i}}, a_{C_{l}, O_{j}}\right)$ are simultaneously satisfied, while $S_{k, l}^{\nu}$ is the number of cases is which $R\left(a_{C_{k}, O_{i}}, a_{C_{k}, O_{j}}\right)$ and $\bar{R}\left(a_{C_{l}, O_{i}}, a_{C_{l}, O_{j}}\right)$ are simultaneously satisfied, it is obvious, that

$$
S_{k, l}^{\mu}+S_{k, l}^{\nu} \leq \frac{n(n-1)}{2} .
$$

Further, for every $k, l$, satisfying $1 \leq k<l \leq m$, and for $n \geq 2$,

$$
\mu_{C_{k}, C_{l}}=2 \frac{S_{k, l}^{\mu}}{n(n-1)}, \quad \nu_{C_{k}, C_{l}}=2 \frac{S_{k, l}^{\nu}}{n(n-1)}
$$

are defined. Therefore, $\left\langle\mu_{C_{k}, C_{l}}, \nu_{C_{k}, C_{l}}\right\rangle$ is an intuitionistic fuzzy pair. Next, the following IM is constructed:

$$
\begin{array}{c|ccc} 
& C_{1} & \ldots & C_{m} \\
\hline C_{1} & \left\langle\mu_{C_{1}, C_{1}}, \nu_{C_{1}, C_{1}}\right\rangle & \ldots & \left\langle\mu_{C_{1}, C_{m}}, \nu_{C_{1}, C_{m}}\right\rangle \\
\vdots & \vdots & \ddots & \vdots \\
C_{m} & \left\langle\mu_{C_{m}, C_{1}}, \nu_{C_{m}, C_{1}}\right\rangle & \ldots & \left\langle\mu_{C_{m}, C_{m}}, \nu_{C_{m}, C_{m}}\right\rangle
\end{array},
$$

that determines the degrees of "correspondence" between criteria $C_{1}, \ldots, C_{m}$.

The sum $\mu_{C_{k}, C_{l}}+\nu_{C_{k}, C_{l}}$ is not always equal to 1 . The difference

$$
\pi_{C_{k}, C_{l}}=1-\mu_{C_{k}, C_{l}}-\nu_{C_{k}, C_{l}}
$$

is considered as a degree of "uncertainty".

The final step of ICrA is to classify the degrees of "correspondence" between criteria. Let $\alpha, \beta \in[0 ; 1]$ are the threshold values for comparison of $\mu_{C_{k}, C_{l}}$ and $\nu_{C_{k}, C_{l}}$. In general, the criteria $C_{k}$ and $C_{l}$ are respectively:

- in a positive consonance, if $\mu_{C_{k}, C_{l}}>\alpha$ and $\nu_{C_{k}, C_{l}}<\beta$; 


\begin{tabular}{c|ccccccc} 
& $O_{1}$ & $\ldots$ & $O_{k}$ & $\ldots$ & $O_{l}$ & $\ldots$ & $O_{n}$ \\
\hline$C_{1}$ & $a_{C_{1}, O_{1}}$ & $\ldots$ & $a_{C_{1}, O_{k}}$ & $\ldots$ & $a_{C_{1}, O_{l}}$ & $\ldots$ & $a_{C_{1}, O_{n}}$ \\
$\vdots$ & $\vdots$ & $\ddots$ & $\vdots$ & $\ddots$ & $\vdots$ & $\ddots$ & $\vdots$ \\
$C_{i}$ & $a_{C_{i}, O_{1}}$ & $\ldots$ & $a_{C_{i}, O_{k}}$ & $\ldots$ & $a_{C_{i}, O_{l}}$ & $\ldots$ & $a_{C_{i}, O_{n}}$ \\
$\vdots$ & $\vdots$ & $\ddots$ & $\vdots$ & $\ddots$ & $\vdots$ & $\ddots$ & $\vdots$ \\
$C_{j}$ & $a_{C_{j}, O_{1}}$ & $\ldots$ & $a_{C_{j}, O_{k}}$ & $\ldots$ & $a_{C_{j}, O_{l}}$ & $\ldots$ & $a_{C_{j}, O_{n}}$ \\
$\vdots$ & $\vdots$ & $\ddots$ & $\vdots$ & $\ddots$ & $\vdots$ & $\ddots$ & $\vdots$ \\
$C_{m}$ & $a_{C_{m}, O_{1}}$ & $\ldots$ & $a_{C_{m}, O_{k}}$ & $\ldots$ & $a_{C_{m}, O_{l}}$ & $\ldots$ & $a_{C_{m}, O_{n}}$
\end{tabular},

- in a negative consonance, if $\mu_{C_{k}, C_{l}}<\beta$ and $\nu_{C_{k}, C_{l}}>\alpha$; - in a dissonance, otherwise.

\section{NUMERICAL RESULTS AND DISCUSSION}

All identification procedures as well as InterCriteria Analysis implementation are performed on PC Intel Pentium 4 (2.4 $\mathrm{GHz}$ ) platform running Windows XP.

Six modifications of MpGA with different execution order of main genetic operators selection, crossover and mutation have been consequently applied to estimate the model parameters (vector $p$ ) of the considered model (1)-(4). Due to the stochastic nature of GA, 30 independent runs for each of the applied here six MpGA have been performed. MpGA operators and parameters are tuned according to [1].

In terms of ICrA, altogether eight criteria are taken into consideration: objective function value $J$ is considered as $C_{1}$; convergence time $T-$ as $C_{2}$; specific growth rates $\mu_{2 S}$ and $\mu_{2 E}$ - respectively as $C_{3}$ and $C_{4}$; saturation constants $k_{S}$ and $k_{E}$ - respectively as $C_{5}$ and $C_{6}$; yield coefficients $Y_{S X}$ and $Y_{E X}-$ respectively as $C_{7}$ and $C_{8}$. Six investigated here objects, referred to MpGA modifications, respectively are: $O_{1}$ corresponding to MpGA-CMS; $\mathrm{O}_{2}$ - to MpGA-CSM; $\mathrm{O}_{3}$ - to MpGA-MCS; $O_{4}$ - to MpGA-MSC; $O_{5}$ - to MpGASCM; and $O_{6}$ - to MpGA-SMC. For convenience, forenames of objective function, convergence time, fermentation process model parameters and MpGA modifications are further used instead of a criterion $C_{i}$ or an object $O_{j}$.

IMs (10)-(12) present, respectively, the average estimates (10), the best ones (11), and the worst ones (12) of the values of objective function $J$, the algorithm convergence time $T$, [s], as well as of all model parameters towards vector $p$. Three IMs list the objective function values rounded to the fifth digit after the decimal point, while the rest criteria - to the fourth digit after the decimal point. However, at the step of ICrA implementation, all parameter estimates are used as they have been obtained as a result from parameter identification procedures, in order to be distinguishable and the degrees of "uncertainty" to be decreased.

As seen from (10)-(12), obtained results show similar values for objective function $J$ after application of the considered here six MpGA for fermentation process model parameter identification. There is about $1 \%$ difference between the best among the best results $(J=0.02193$ for MpGA-SMC, (11) and the worst among the worst results $(J=0.02218$ for
MpGA-CSM, (12)). On the other hand, the convergence time $T$ increases more than 2.5 times (347.3300 for MpGA-CMS, (12) towards 131.4000 for MpGA-SCM, (11)). Such a small deviation of $J$ proves all six considered here MpGA modifications as equally reliable and it is of user choice to make a compromise between the model accuracy and convergence time.

ICrA approach has been consequently implemented for each of the constructed IMs $A_{1}$ (average), $A_{2}$ (best) and $A_{3}$ (worst). After ICrA application, six IMs that determine the degrees of agreement and disagreement between investigated criteria have been obtained. IMs themselves are not shown here, but the results from the ICrA implementation for the cases of average, best and worst evaluations have been summarized in Table I. Obtained results are ranked by $\mu_{C_{k} ; C_{l}}$ values in the case of average evaluations. As could be seen from Table I, there are no pairs with a degree of "uncertainty" for the cases of average and worst evaluations, while such criteria pairs have been observed in the case of the best evaluations. The logical explanation of this fact is that even using a "row data" from parameter identification procedures there are some equal evaluations for some of the model parameters in different MpGA.

Aiming at better interpretation of the obtained results listed above, they are also graphically presented in Fig. 1.

Table II presents the scale of consonance and dissonance [17], on which basis each pair of criteria is going to be assessed.

Based on the presented scale, the following pair dependencies might be outlined for the case of average results of the examined criteria.

A positive and a weak positive consonance have been observed respectively for the pairs $\mu_{2 E}-Y_{E X}$ and $k_{S}-Y_{E X}$. There are three criteria pairs in a negative consonance - $J-k_{E}$, $Y_{S X}-Y_{E X}$ and $\mu_{2 E}-Y_{S X}$ and another one $-\mu_{2 S}-k_{S}$ in a weak negative consonance. The rest of the criteria pairs hit the intervals of a dissonance.

Some coincidences for the three investigated here case studies (of average, best and worst results) should be mentioned. The criteria pair $\mu_{2 E}-Y_{E X}$ is in a positive consonance for the cases of average and best results. The same criteria pair hits the upper boundary of the $\mu$-values in the case of worst results, as such showing a strong positive consonance. The criteria pair $\mu_{2 E}-Y_{E X}$ is the only one with the maximum value (i.e. $\mu=1$ ) for the degree of "agreement". A negative consonance for the pair $\mu_{2 E}-Y_{S X}$ has been observed for 


\begin{tabular}{c|c|c|c|c|c|c} 
& MpGA-CMS & MpGA-CSM & MpGA-MCS & MpGA-MSC & MpGA-SCM & MpGA-SMC \\
\hline$J$ & 0.02203 & 0.02210 & 0.02196 & 0.02203 & 0.02206 & 0.02195 \\
$T$ & 370.9408 & 317.6379 & 265.4929 & 214.0625 & 113.9127 & 164.3988 \\
$\mu_{2 S}$ & 0.9002 & 0.9063 & 0.9076 & 0.9023 & 0.9018 & 0.9004 \\
$A_{1}$ (average $)=\mu_{2 E}$ & 0.1494 & 0.1242 & 0.1485 & 0.1216 & 0.1389 & 0.1484 \\
$k_{S}$ & 0.1500 & 0.1496 & 0.1487 & 0.1466 & 0.1500 & 0.1500 \\
$k_{E}$ & 0.8000 & 0.8000 & 0.8339 & 0.8366 & 0.8000 & 0.8420 \\
$Y_{S X}$ & 0.3973 & 0.4090 & 0.3960 & 0.4126 & 0.4022 & 0.3993 \\
$Y_{E X}$ & 2.0193 & 1.6524 & 1.9939 & 1.6035 & 1.8630 & 2.0019
\end{tabular}

\begin{tabular}{c|c|c|c|c|c|c} 
& MpGA-CMS & MpGA-CSM & MpGA-MCS & MpGA-MSC & MpGA-SCM & MpGA-SMC \\
\hline$J$ & 0.02203 & 0.02204 & 0.02195 & 0.02199 & 0.02203 & 0.02193 \\
$T$ & 377.9200 & 343.0900 & 288.7900 & 226.7900 & 131.4000 & 177.1900 \\
$\mu_{2 S}$ & 0.9000 & 0.9004 & 0.9009 & 0.9024 & 0.9000 & 0.9004 \\
$A_{2}$ (best $)=\mu_{2 E}$ & 0.1500 & 0.1444 & 0.1496 & 0.1244 & 0.1500 & 0.1484 \\
$k_{S}$ & 0.1500 & 0.1500 & 0.1500 & 0.1465 & 0.1500 & 0.1500 \\
$k_{E}$ & 0.8000 & 0.8000 & 0.8363 & 0.8491 & 0.8000 & 0.8509 \\
$Y_{S X}$ & 0.3969 & 0.3998 & 0.3952 & 0.4120 & 0.3975 & 0.3993 \\
$Y_{E X}$ & 2.0297 & 1.9453 & 2.0009 & 1.6398 & 2.0280 & 2.0020
\end{tabular}

\begin{tabular}{c|c|c|c|c|c|c} 
& MpGA-CMS & MpGA-CSM & MpGA-MCS & MpGA-MSC & MpGA-SCM & MpGA-SMC \\
\hline$J$ & 0.02204 & 0.02218 & 0.02198 & 0.02205 & 0.02211 & 0.02201 \\
$T$ & 347.3300 & 295.4300 & 253.5500 & 195.9700 & 92.2960 & 142.9400 \\
$\mu_{2 S}$ & 0.9044 & 0.9176 & 0.9248 & 0.9024 & 0.9220 & 0.9014 \\
$A_{3}$ (worst $)=\mu_{2 E}$ & 0.1460 & 0.1003 & 0.1472 & 0.1148 & 0.1211 & 0.1480 \\
$k_{S}$ & 0.1498 & 0.1488 & 0.1455 & 0.1466 & 0.1495 & 0.1500 \\
$k_{E}$ & 0.8000 & 0.8000 & 0.8359 & 0.8413 & 0.8000 & 0.8095 \\
$Y_{S X}$ & 0.4006 & 0.4196 & 0.3959 & 0.4143 & 0.4103 & 0.3992 \\
$Y_{E X}$ & 1.9660 & 1.3112 & 1.9771 & 1.5232 & 1.6125 & 2.0002
\end{tabular}

the three considered case studies. A negative consonance has been outlined for $Y_{S X}-Y_{E X}$ in the cases of average and worst results, while in the case of the best results the pair is in a weak negative consonance.

Although mentioned above coincidences, there are several discrepancies in criteria dependences, caused mainly by the stochastic nature of the considered here six modifications of MpGA. The attention is drawn only for the pairs, showing consonance in some of the considered case studies. For example, according to the scale presented in Table II, a weak positive consonance has been observed for criteria pair $k_{S}-Y_{E X}$ in the cases of average and best results, while in the case of the worst results the pair is in a dissonance. A weak positive consonance for $\mu_{2 E}-k_{S}$ and a positive consonance for $J-Y_{S X}$ could be seen only in the cases of best and worst results, respectively. Another discrepancy is found in the results for the criteria pairs $\mu_{2 S}-k_{S}$ and $J-k_{E}$. Both pairs are in a negative consonance for the case studies of average and best results, while in the case of the worst results they fall in the interval of a weak dissonance.

Distribution of dependencies between criteria pairs in the cases of average, best and worst evaluations are listed in Table III. The criteria pairs are equally distributed in the cases of average and worst results. In the case of the best results, there are more criteria pairs in a positive or in a negative consonance and less in a dissonance, but this is the only one case with observed degree of "uncertainty" for some of the criteria pairs.

Taking into account the obtained ICrA estimations in the cases of average, best and worst results, and having in mind the stochastic nature of GA, it is more reasonable to rely with a higher credibility on the results in the case of average values than to results obtained in another two cases.

\section{CONCLUSion}

Promising ICrA approach has been here implemented to examine the performance of six modifications of multipopulation genetic algorithms, applied for the purposes of a fermentation process model parameter identification. All considered MpGA modifications demonstrate almost equal degree of accuracy with about $1 \%$ objective function value divergence, but on the account of the convergence time. Thus, it is of user choice to make a compromise between model accuracy and convergence time. After applying MpGA to a parameter identification of $S$. cerevisiae fed-batch cultivation process, three case studies have been examined - of average, 
TABLE I

CRITERIA RELATIONS SORTED BY $\mu_{C_{k}, C_{l}}$ VALUES IN THE CASE OF AVERAGE RESUlTS

\begin{tabular}{c|c|c|c}
\hline \multirow{2}{*}{ Criteria relation } & Average results & Best results & Worst results \\
\cline { 2 - 4 } & $\mu / \nu / \pi$ & $\mu / \nu / \pi$ & $\mu / \nu / \pi$ \\
\hline$\mu_{2 E}-Y_{E X}$ & $0.93 / 0.07 / 0.00$ & $0.87 / 0.07 / 0.06$ & $1.00 / 0.00 / 0.00$ \\
\hline$k_{S}-Y_{E X}$ & $0.80 / 0.20 / 0.00$ & $0.80 / 0.00 / 0.20$ & $0.67 / 0.33 / 0.00$ \\
\hline$\mu_{2 E}-k_{S}$ & $0.73 / 0.27 / 0.00$ & $0.80 / 0.07 / 0.13$ & $0.67 / 0.33 / 0.00$ \\
\hline$J-Y_{S X}$ & $0.67 / 0.33 / 0.00$ & $0.60 / 0.40 / 0.00$ & $0.93 / 0.07 / 0.00$ \\
\hline$T-\mu_{2 E}$ & $0.67 / 0.33 / 0.00$ & $0.47 / 0.47 / 0.06$ & $0.40 / 0.60 / 0.00$ \\
\hline$J-T$ & $0.60 / 0.40 / 0.00$ & $0.60 / 0.40 / 0.00$ & $0.47 / 0.53 / 0.00$ \\
\hline$T-Y_{E X}$ & $0.60 / 0.40 / 0.00$ & $0.47 / 0.53 / 0.00$ & $0.40 / 0.60 / 0.00$ \\
\hline$\mu_{2 S}-Y_{S X}$ & $0.60 / 0.40 / 0.00$ & $0.73 / 0.27 / 0.00$ & $0.47 / 0.53 / 0.00$ \\
\hline$J-\mu_{2 S}$ & $0.53 / 0.47 / 0.00$ & $0.47 / 0.53 / 0.00$ & $0.53 / 0.47 / 0.00$ \\
\hline$T-\mu_{2 S}$ & $0.53 / 0.47 / 0.00$ & $0.47 / 0.53 / 0.00$ & $0.53 / 0.47 / 0.00$ \\
\hline$T-k_{S}$ & $0.53 / 0.47 / 0.00$ & $0.33 / 0.47 / 0.20$ & $0.47 / 0.53 / 0.00$ \\
\hline$k_{E}-Y_{E X}$ & $0.53 / 0.47 / 0.00$ & $0.40 / 0.53 / 0.07$ & $0.60 / 0.40 / 0.00$ \\
\hline$\mu_{2 S}-k_{E}$ & $0.47 / 0.53 / 0.00$ & $0.60 / 0.33 / 0.07$ & $0.47 / 0.53 / 0.00$ \\
\hline$\mu_{2 E}-k_{E}$ & $0.47 / 0.53 / 0.00$ & $0.40 / 0.60 / 0.00$ & $0.60 / 0.40 / 0.00$ \\
\hline$k_{S}-k_{E}$ & $0.47 / 0.53 / 0.00$ & $0.47 / 0.40 / 0.13$ & $0.40 / 0.60 / 0.00$ \\
\hline$k_{E}-Y_{S X}$ & $0.47 / 0.53 / 0.00$ & $0.47 / 0.47 / 0.06$ & $0.33 / 0.67 / 0.00$ \\
\hline$J-\mu_{2 E}$ & $0.40 / 0.60 / 0.00$ & $0.53 / 0.40 / 0.07$ & $0.13 / 0.87 / 0.00$ \\
\hline$J-k_{S}$ & $0.40 / 0.60 / 0.00$ & $0.33 / 0.47 / 0.20$ & $0.47 / 0.53 / 0.00$ \\
\hline$T-Y_{S X}$ & $0.40 / 0.60 / 0.00$ & $0.47 / 0.53 / 0.00$ & $0.53 / 0.47 / 0.00$ \\
\hline$J-Y_{E X}$ & $0.33 / 0.67 / 0.00$ & $0.47 / 0.53 / 0.00$ & $0.13 / 0.87 / 0.00$ \\
\hline$\mu_{2 S}-\mu_{2 E}$ & $0.33 / 0.67 / 0.00$ & $0.13 / 0.80 / 0.07$ & $0.47 / 0.53 / 0.00$ \\
\hline$k_{S}-Y_{S X}$ & $0.33 / 0.67 / 0.00$ & $0.20 / 0.60 / 0.20$ & $0.40 / 0.60 / 0.00$ \\
\hline$T-k_{E}$ & $0.27 / 0.73 / 0.00$ & $0.27 / 0.67 / 0.06$ & $0.40 / 0.60 / 0.00$ \\
\hline$\mu_{2 S}-Y_{E X}$ & $0.27 / 0.73 / 0.00$ & $0.07 / 0.93 / 0.00$ & $0.47 / 0.53 / 0.00$ \\
\hline$\mu_{2 S}-k_{S}$ & $0.20 / 0.80 / 0.00$ & $0.07 / 0.73 / 0.20$ & $0.27 / 0.73 / 0.00$ \\
\hline$J-k_{E}$ & $0.13 / 0.87 / 0.00$ & $0.07 / 0.87 / 0.06$ & $0.27 / 0.73 / 0.00$ \\
\hline$Y_{S X}-Y_{E X}$ & $0.13 / 0.87 / 0.00$ & $0.20 / 0.80 / 0.00$ & $0.07 / 0.93 / 0.00$ \\
\hline$\mu_{2 E}-Y_{S X}$ & $0.07 / 0.93 / 0.00$ & $0.13 / 0.80 / 0.07$ & $0.07 / 0.93 / 0.00$ \\
\hline & & &
\end{tabular}

TABLE II

SCALE OF CONSONANCE AND DISSONANCE

\begin{tabular}{c|l}
\hline Interval of $\mu_{C_{k}, C_{l}}$ & Meaning \\
\hline$[0.00-0.05]$ & strong negative consonance \\
\hline$(0.05-0.15]$ & negative consonance \\
\hline$(0.15-0.25]$ & weak negative consonance \\
\hline$(0.25-0.33]$ & weak dissonance \\
\hline$(0.33-0.43]$ & dissonance \\
\hline$(0.43-0.57]$ & strong dissonance \\
\hline$(0.57-0.67]$ & dissonance \\
\hline$(0.67-0.75]$ & weak dissonance \\
\hline$(0.75-0.85]$ & weak positive consonance \\
\hline$(0.85-0.95]$ & positive consonance \\
\hline$(0.95-1.00]$ & strong positive consonance \\
\hline
\end{tabular}

best and worst results in regard to chosen criteria. ICrA approach has been implemented to assists in establishing of
TABLE III

DISTRIBUTION OF DEPENDENCES BETWEEN CRITERIA PAIRS

\begin{tabular}{c|c|c|c}
\hline Meaning & Average & Best & Worst \\
\hline Positive consonance & 2 & 3 & 2 \\
\hline Dissonance & 22 & 18 & 22 \\
\hline Negative consonance & 4 & 7 & 4 \\
\hline
\end{tabular}

existing relations between fermentation process model parameters and MpGA outcomes, such as objective function value and convergence time. Obtained additional knowledge for relations between model parameters and algorithms outcomes might be useful for improving the model accuracy and the performance of optimization algorithms in further parameter identification procedures.

\section{ACKNOWLEDGEMENT}

The work is partially supported by the National Science Fund of Bulgaria under the grant DFNI-I-02-5 "InterCriteria 

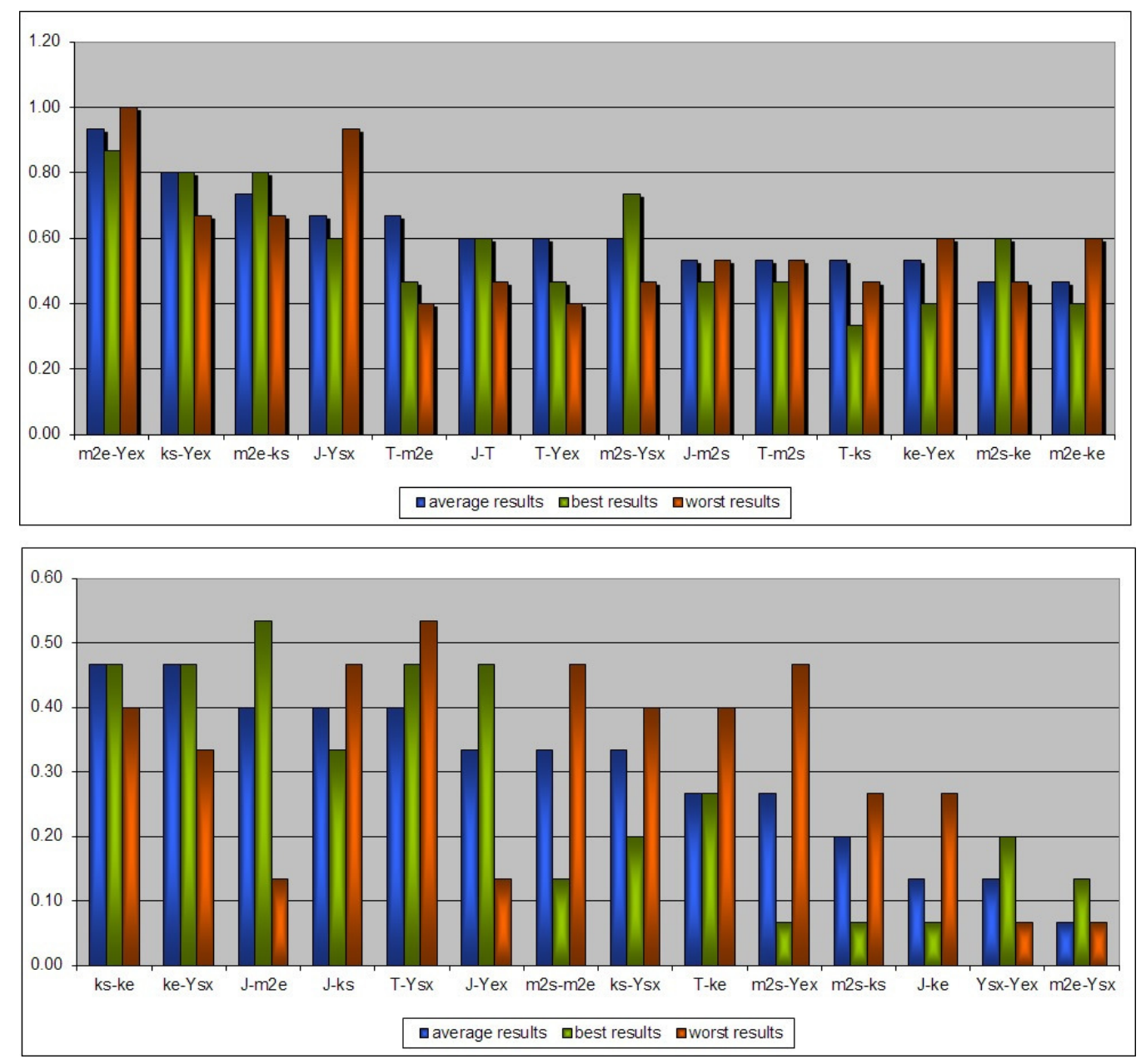

Fig. 1. Degrees of "agreement" $\left(\mu_{C_{k}, C_{l}}\right.$ values) for three considered cases

Analysis - A New Approach to Decision Making" and DM07/1 "Development of New Modified and Hybrid Metaheuristic Algorithms".

\section{REFERENCES}

[1] M. Angelova and T. Pencheva, "Genetic operators' significance assessment in multi-population genetic algorithms", Int. J. of Metaheuristics, vol. 3(2), 2014, pp. 162-173, DOI: 10.1504/IJMHEUR.2014.063146.

[2] M. Angelova, O. Roeva and T. Pencheva, "Intercriteria analysis of crossover and mutation rates relations in simple genetic algorithm", Ann. Comp. Sci. Info. Syst., vol. 5, 2015, pp. 419-424, DOI: 10.15439/2015F178.

[3] M. Angelova, S. Tzonkov and T. Pencheva,"Modified multi-population genetic algorithm for yeast fed-batch cultivation parameter identification", Int. J. Bioautomation, vol. 13(4), 2009, pp. 163-172.

[4] K. Atanassov, On intuitionistic fuzzy sets theory, Springer, Berlin, 2012, DOI: $10.1007 / 978-3-642-29127-2$.

[5] K. Atanassov, "Generalized index matrices", C. R. Acad. Bulg. Sci., vol. 40(11), 1987, pp. 15-18.

[6] K. Atanassov, "On index matrices, part 1: standard cases", Adv. Stud. Cont. Math., vol. 20(2), 2010, pp. 291-302.

[7] K. Atanassov, "On index matrices, part 2: intuitionistic fuzzy case", Proc. Jangjeon Math. Soc., vol. 13(2), 2010, pp. 121-126.

[8] K. Atanassov, D. Mavrov and V. Atanassova, "Intercriteria decision making: a new approach for multicriteria decision making, based on index matrices and intuitionistic fuzzy sets", Iss. Int. Fuz. Sets and Gen. Nets, vol. 11, 2014, pp. 1-8.
[9] A. Ghaheri, S. Shoar, M. Naderan and S. S. Hoseini, "The applications of genetic algorithms in medicine", Oman Med. J., vol. 30(6), 2015, pp. 406-416, DOI: $10.5001 /$ omj.2015.82.

[10] D. E. Goldberg, Genetic algorithms in search, optimization and machine learning, Addison Wesley Longman, London, 2006.

[11] J. Holland, Adaptation in natural and artificial systems: an introductory analysis with application to biology, control and artificial intelligence, University of Michigan Press, 1975.

[12] T. Ilkova and M. Petrov, "Intercriteria analysis for identification of Escherichia coli fed-batch mathematical model", J. Int. Sci. Publ.: Mat., Meth. \& Techn., vol. 9, 2015, pp. 598-608.

[13] T. Pencheva and M. Angelova, "Intercriteria analysis of simple genetic algorithms performance", Advanced Computing in Industrial Mathematics, Vol. 681 of Studies in Computational Intelligence, 2017, 147-159. DOI: 10.1007/978-3-319-49544-6_13.

[14] T. Pencheva and M. Angelova, "Modified multi-population genetic algorithms for parameter identification of yeast fed-batch cultivation", Bulg. Chem. Comm., vol. 48(4), 2016, pp. 713-719.

[15] T. Pencheva, O. Roeva and I. Hristozov, Functional state approach to fermentation processes modelling, Prof. M. Drinov Acad. Publ. House, Sofia, 2006.

[16] O. Roeva (Ed.), Real-world application of genetic algorithms, InTech, 2012, DOI: $10.5772 / 2674$.

[17] O. Roeva, S. Fidanova, P. Vassilev and P. Gepner, "Intercriteria analysis of a model parameters identification using genetic algorithm", Ann. Comp. Sci. Inf. Syst., vol. 5, 2015, pp. 501-506, DOI: $10.15439 / 2015 \mathrm{~F} 223$. 